NOUVELLE

\title{
Pax3 et Pax7 : gardiens du développement craniofacial
}

Antoine Zalc ${ }^{1-3}$, Frédéric Relaix ${ }^{1-5}$

Exposition à la dioxine

et malformations craniofaciales

L'exposition in utero de l'embryon à des polluants environnementaux tératogènes peut conduire à des altérations importantes du développement [1]. Un tératogène est défini comme un composant qui, lorsqu'une femme enceinte y est exposée, est susceptible de provoquer des malformations chez l'enfant. Parmi les tératogènes d'origine environnementale, la dioxine a été particulièrement médiatisée suite aux expositions à grande échelle de populations pendant la guerre du Vietnam (utilisation de l'agent Orange, un herbicide défoliant) ou, plus récemment, lors de l'accident industriel de Seveso en Italie (contaminant une zone de $15 \mathrm{~km}^{2}$ et ses 37000 habitants). L'existence d'une association entre ces expositions à la dioxine et une augmentation du nombre d'anomalies du développement, notamment craniofacial, chez l'humain a été suggérée mais jamais rigoureusement démontrée. Expérimentalement chez la souris, l'exposition à la dioxine in utero génère des fentes palatines [2]. Le mécanisme d'action de ces tératogènes n'est pas connu, mais il a été proposé qu'ils pourraient interférer avec les voies génétiques contrôlant les processus de développement [3].

Chez les vertébrés, la plus grande partie de la face dérive des cellules de la crête neurale. Cette structure embryonnaire transitoire est composée de cellules multipotentes qui, après leur formation à la frontière entre l'ectoderme de surface et la plaque neurale pendant la fermeture du tube neural, migrent à travers l'embryon dans une direction dorso- ventrale en suivant des flux migratoires stéréotypés, puis s'établissent dans des structures embryonnaires spécifiques avant de donner naissance à une grande variété de types cellulaires. Ces cellules donnent notamment naissance à tout le système nerveux périphérique, mais aussi aux cellules pigmentées. Dans la partie la plus rostrale de l'embryon, les cellules émigrant depuis le diencéphale et le mésencéphale antérieur, appelées cellules de la crête neurale céphalique, génèrent des cellules nerveuses, mais forment également la majorité du mésenchyme, des os et du cartilage de la face ainsi que les tendons et les tissus conjonctifs [4].

\section{Implication des gènes $\operatorname{Pax} 3$ et $P a x 7$} pendant la formation de la face Pendant les différentes étapes de l'embryogenèse, le développement des cellules issues de la crête neurale est très finement régulé par un vaste réseau génétique fortement conservé chez tous les vertébrés [3]. Les gènes paralogues Pax3 et Pax7, codant pour des facteurs de transcription à homéoboîte caractérisés par un domaine de liaison à l'ADN dénommé paired-box, y jouent un rôle central en intégrant différents signaux afin d'assurer un développement normal [3]. En effet, chez l'amphibien et le poulet, Pax3 et Pax7 sont requis pour l'induction et la formation de la crête neurale [5-7]. Chez le poisson zèbre, l'absence de Pax3 entraîne des défauts de différenciation de cellules pigmentaires et leur disparition complète au niveau du tronc [8]. Chez l'homme, les mutations hétérozygotes du gène PAX3 sont liées aux syndromes de Waar-
${ }^{1}$ Sorbonne Universités,

Université Pierre et Marie Curie,

Université Paris 06, Inserm U974, CNRS FRE 3617, Institut de myologie, 47, boulevard de l'Hôpital, 75013 Paris, France ;

${ }^{2}$ Inserm, Institut Mondor de recherches biomédicales, U955-E10, 94000 ,

Créteil, France ;

${ }^{3}$ Université Paris Est-Créteil,

Faculté de médecine, F-94000 Créteil,

France ;

4 Établissement français du sang, 94017 Créteil, France ;

${ }^{5}$ Université Paris Est,

École nationale vétérinaire d'Alfort, 94700 Maisons-Alfort, France.

frederic.relaix@inserm.fr

denburg de type I et III, caractérisés par des déficiences auditives, pigmentaires et des défauts craniofaciaux (OMIM \#193500 et OMIM\#148820).

Chez l'embryon de souris, entre les jours $8,5(\varepsilon 8,5)$ et $\varepsilon 13,5$ de gestation, l'expression de Pax 3 et Pax 7 est particulièrement dynamique dans les structures craniofaciales. Bien que la fonction de Pax3 et Pax7 pendant le développement précoce de la crête neurale soit bien caractérisée et remarquablement conservée chez les vertébrés, le rôle de ces facteurs durant le développement craniofacial tardif n'a pas été étudié. Alors que chez les simples mutants Pax 3 ou Pax7 le développement craniofacial est quasiment normal, des mutants chez lesquels les fonctions des deux facteurs sont perturbées présentent des phénotypes craniofaciaux sévères. Le double mutant Pax3:Pax7, ainsi qu'un modèle dominant négatif de Pax3 [9], présentent tous les deux une dysplasie faciale sévère, le phénotype étant exacerbé chez le double mutant. Nous avons développé un modèle dominant négatif, dans lequel un des allèles de Pax 3 est substitué par une séquence codant pour la GFP (green fluorescent protein) alors que le second allèle est remplacé par une séquence codant pour une protéine de fusion entre la partie amino-terminale de Pax3, possédant les domaines de 


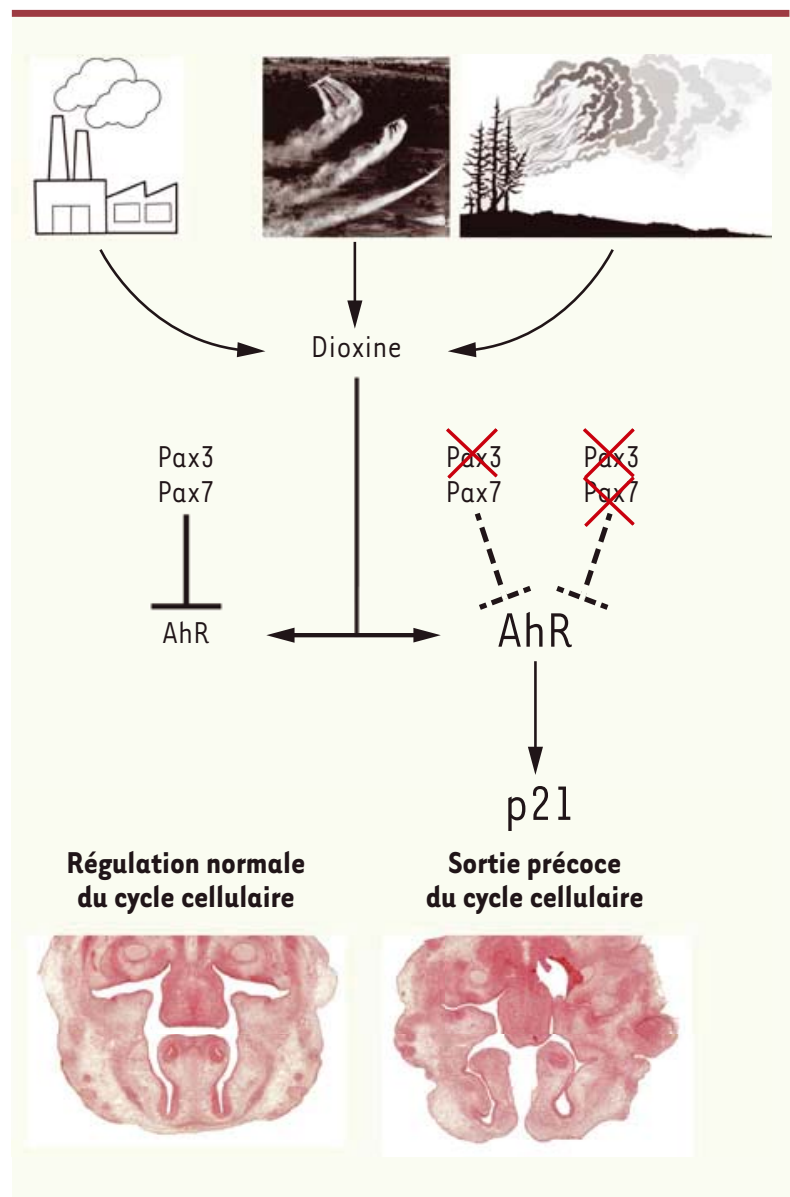

Figure 1. Interaction entre la voie AhR et le développement craniofacial. Les photos représentent différentes sources pouvant libérer de la dioxine dans l'environnement tels que les incinérateurs industriels et municipaux, les épandages de produits chimiques ou herbicides et les causes naturelles que sont les feux de forêt ou les éruptions volcaniques. Lorsque l'embryon est exposé à la dioxine, dans les conditions normales, Pax3 et Pax7 protègent son développement en inhibant l'expression et l'action d'AhR. Cependant, en absence de Pax3 ou lorsque la fonction de Pax3 et Pax7 est inhibée, plus rien ne s'oppose à une forte activation de la voie AhR par la présence de dioxine dans l'organisme. Ceci déclenche une sortie précoce du cycle cellulaire des cellules dérivées des crêtes neurales céphaliques via une surexpression de p21, et entraîne la formation d'une fente oro-faciale chez les embryons mutants.

Une voie de réponse au stress environnemental régule la croissance embryonnaire de la face

Afin d'établir les réseaux génétiques contrôlés par Pax3 et $\mathrm{Pax} 7$ pendant le développement de la face, nous avons combiné une

liaisons à l'ADN, et le domaine répresseur de la transcription de la protéine Engrailed [9]. Dans ce modèle, bien que Pax7 soit toujours exprimé, sa fonction, ainsi que celle de Pax3, sont inhibées. L'étude des phénotypes de ces différents mutants démontre que $\mathrm{Pax} 3$ et $\mathrm{Pax} 7$ jouent des rôles cruciaux et redondants pendant le développement craniofacial. Nous avons montré que l'absence de Pax3 et Pax7 entraîne une induction significative de l'apoptose des cellules issues des crêtes neurales chez le double mutant, expliquant le phénotype observé. Cet effet drastique détecté chez les doubles mutants ne permet pas l'étude des mécanismes cellulaires et moléculaires mis en cause, au contraire de notre modèle dominant-négatif. Dans ce modèle, en effet, l'apoptose cellulaire n'est pas modifiée, ce qui nous a permis d'étudier les réseaux génétiques intervenant en aval de ces facteurs dans le développement craniofacial. approche de tri en cytométrie de flux avec des analyses en puces à ADN. Nous avons comparé les transcriptomes des cellules issues des crêtes neurales de notre modèle dominant négatif et d'un contrôle [10]. Cette comparaison a mis en évidence la surexpression de la voie de signalisation régulée par le AhR (aryl hydrocarbon receptor), le récepteur de la dioxine, chez le dominant négatif. La capacité du récepteur AhR à lier la dioxine en a fait un acteur majeur de la réponse au stress environnemental [11]. Dans notre modèle où les fonctions de Pax3 et Pax7 sont inhibées, nous avons observé que l'augmentation de l'activité d'AhR induit précocement la sortie du cycle cellulaire des cellules mésenchymateuses de la face via la surexpression de l'inhibiteur de kinases cycline-dépendantes p21. Cette sortie prématurée du cycle cellulaire conduit à une réduction du nombre de cellules progénitrices dans les structures nasales. II en résulte une croissance réduite de ces structures et une absence de fusion de la face, qui explique la fente oro-faciale frontale observée chez le fœtus mutant pour Pax3 et Pax7 (Figure 1).

Afin de démontrer le rôle inédit de la voie de signalisation AhR dans la régulation du cycle cellulaire des cellules issues de la crête neurale, nous avons montré, in vitro et in vivo, que l'inhibition de l'activité d'AhR est suffisante pour restaurer la prolifération de ces cellules chez notre modèle dominant négatif via une normalisation des niveaux d'expression de p21, permettant ainsi la fermeture de la face. Nos résultats démontrent ainsi qu'une interaction entre une voie de signalisation impliquée dans la réponse au stress environnemental et les voies moléculaires contrôlées par Pax3 et Pax7 est nécessaire pour un développement craniofacial normal [10].

\section{Une nouvelle fonction pour Pax3 et $\operatorname{Pax} 7$ pendant le développement craniofacial}

Lorsque des embryons mutants pour Pax3, qui ne présentent pas de défaut craniofacial, sont exposés à des doses non tératogènes de dioxine, nous avons observé l'apparition d'une fente orofaciale frontale (les embryons hétérozygotes se développant normalement) confortant ainsi l'hypothèse selon laquelle les gènes Pax 3 et Pax 7 régulent la croissance et le maintien des cellules issues des crêtes neurales via la 
voie de signalisation AhR. Cette survenue de défauts craniofaciaux chez les simples mutants Pax3 confirme le rôle protecteur joué par les gènes Pax 3 et Pax7 vis-à-vis de polluants tératogènes pendant le développement craniofacial. De plus, cette observation conforte nos précédentes conclusions établissant la voie AhR comme un médiateur essentiel de l'action des gènes Pax3 et Pax7 dans la régulation du développement des cellules issues de la crête neurale pendant la morphogenèse craniofaciale [10]. $\diamond$ Pax3 and Pax7 play essential safeguard functions against environmental stress-induced birth defects

\section{LIENS D'INTÉRÊT}

Les auteurs déclarent n'avoir aucun lien d'intérêt concernant les données publiées dans cet article.

\section{RéFÉRENCES}

1. Gilbert-Barness $\varepsilon$. Teratogenic causes of malformations. Ann Clin Lab Sci $2010 ; 40$ : 99-114.

2. Courtney KD, Moore JA. Teratology studies with 2,4,5-trichlorophenoxyacetic acid and 2,3,7,8-tetrachlorodibenzo-p-dioxin. Toxicol Appl Pharmacol $1971 ; 20$ : 396-403.

3. Betancur P, Bronner-Fraser M, Sauka-Spengler T. Assembling neural crest regulatory circuits into a gene regulatory network. Annu Rev Cell Dev Biol 2010 ; $26: 581-603$.

4. Le Douarin N, Kalcheim C. The neural crest. Cambridge : Cambridge University Press, 1999.

5. Basch ML, Bronner-Fraser M, Garcia-Castro MI. Specification of the neural crest occurs during gastrulation and requires Pax7. Nature 2006 ; 441 : 218-22.
6. Monsoro-Burq AH, Wang E, Harland R. Msxl and Pax3 cooperate to mediate FGF8 and WNT signals during Xenopus neural crest induction. Dev Cell 2005 ; 8 : $167-78$.

7. Sato T, Sasai N, Sasai Y. Neural crest determination by co-activation of Pax3 and Zicl genes in Xenopus ectoderm. Development 2005 ; 132 : 2355-63.

8. Minchin JE, Hughes SM. Sequential actions of Pax 3 and Pax7 drive xanthophore development in zebrafish neural crest. Dev Biol 2008 ; 317 : 508-22.

9. Bajard L, Relaix F, Lagha M, et al. A novel genetic hierarchy functions during hypaxial myogenesis: Pax3 directly activates Myf5 in muscle progenitor cells in the limb. Genes Dev 2006; $20: 2450-64$.

10. Zalc A, Rattenbach R, Auradé F, et al. Pax3 and Pax7 play essential safeguard functions against environmental stress-induced birth defect. Dev Cell $2015 ; 33: 56-66$.

11. Fernandez-Salguero PM, Hilbert DM, Rudikoff S, et al. Aryl-hydrocarbon receptor-deficient mice are resistant to 2,3,7,8-tetrachlorodibenzo-p-dioxininduced toxicity. Toxicol Appl Pharmacol 1996 ; 140 : $173-9$.

\section{NOUVELLE}

\section{Origine et développement des cellules dendritiques humaines}

Gaëlle Breton

Laboratory of cellular physiology and immunology, the Rockefeller University, 1230 York avenue, 10065 New York, Etats-Unis.

gbreton@rockefeller.edu
> Les cellules dendritiques ont été découvertes en 1973 par Ralph Steinman et Zanvil Cohn alors que ces derniers cherchaient à comprendre comment une réponse immunitaire était induite dans les rates de souris [11]. Ils savaient que pour qu'une réponse immunitaire se développe, il fallait des lymphocytes mais aussi une cellule « accessoire » présentatrice d'antigènes. À cette époque, les immunologistes pensaient que cette cellule accessoire était un macrophage, et ce jusqu'à ce que R. Steinman et Z. Cohn découvrent une population de cellules rares, jamais observées auparavant, et à la forme inhabituelle; parce que cette cellule était constellée de longs prolongements arborescents, R. Steinman et Z. Cohn l'ont appelée cellule dendritique (du grec dendreon qui signifie arbre). R. Steinman et Z. Cohn ont eu l'intuition que cette cellule était la cellule accessoire qu'ils recherchaient $[1,2]$. Néanmoins, il leur a fallu beaucoup d'efforts et de persévérance pour démontrer que les cellules dendritiques n'étaient pas des macrophages mais bel et bien un type cellulaire différent, et en convaincre leurs collègues immunologistes [3]. Ce n'est que trois décennies plus tard que l'origine des cellules dendritiques chez la souris a été élucidée, et que le point de divergence entre les cellules dendritiques et les monocytes/ macrophages a été défini [4].

\section{Les différentes sous-populations} de cellules dendritiques humaines Les cellules dendritiques humaines ont été principalement étudiées dans le sang périphérique, et ont été classées en sous-populations en se fondant sur leur fonction et l'expression de marqueurs de surface. Les cellules dendritiques plasmacytoïdes expriment CD303 (BDCA-2, blood dendritic cell antigen 2) et ont l'unique capacité de produire de larges quantités d'interférons de type I en réponse à une infection virale [5]. Les cellules dendritiques myéloïdes - dites conventionnelles - qui expriment CDlc (BDCA-1) - présenteraient préférentiellement les antigènes aux cellules $T$ $\mathrm{CD}^{+}$et seraient l'équivalent des cellules dendritiques $\mathrm{CD} 1 \mathrm{lb}^{+}$chez la souris $[6,7]$. Enfin, les cellules dendritiques conventionnelles qui expriment CD141 (BDCA-3) sont équivalentes aux cellules dendritiques CD8 $\alpha^{+}$murines ; elles ont la capacité de capturer les cellules nécrotiques 